\title{
Articulated Motion and Deformable Objects
}

\author{
Jun $\mathrm{Wan}^{a}$, Sergio Escalera ${ }^{b}$, Francisco J. Perales ${ }^{c}$, Josef Kittler ${ }^{d}$ \\ a National Laboratory of Pattern Recognition, Institute of Automation, Chinese Academy of \\ Sciences, China \\ ${ }^{b}$ University of Barcelona and Computer Vision Center, Spain \\ ${ }^{c}$ Computer Graphics, Vision and Artificial Intelligence Group, Universitat de les Illes \\ Balears, Spain \\ ${ }^{d}$ Centre for Vision, Speech and Signal Processing, University of Surrey, UK
}

\begin{abstract}
This guest editorial introduces the twenty papers accepted for this Special Issue on Articulated Motion and Deformable Objects (AMDO). They are grouped into four main categories within the field of AMDO: human motion analysis (action/gesture), human pose estimation, deformable shape segmentation, and face analysis. For each of the four topics, a survey of the recent developments in the field is presented. The accepted papers are briefly introduced in the context of this survey. They contribute novel methods, algorithms with improved performance as measured on benchmarking datasets, as well as two new datasets for hand action detection and human posture analysis. The special issue should be of high relevance to the reader interested in AMDO recognition and promote future research directions in the field.

Keywords: Articulated Motion and Deformable Objects, Pose Estimation, Action recognition, Gesture recognition, Face Analysis.
\end{abstract}

\section{Introduction}

Articulated motion and deformable objects (AMDO) is a challenging research area which focuses on the automatic analysis of complex objects, such as the human body, exhibiting high variabilities both in terms of spatial and 5 temporal dimensions. AMDO is of high interest in the fields of pattern recog- 
nition, computer vision, computer graphics, biometrics, machine learning and human-computer interface (HCI), to mention just a few.

In the late 2016, contributions to a special issue on AMDO had been invited for possible publication in the Patter Recognition journal by an open call for papers. The scope of the special issue had been defined so as to cover pattern recognition schemes on any AMDO related topics, including human motion analysis and tracking, human reconstruction, multimodal AMDO, 2D/3D deformable models, and new pattern recognition applications in the field of AMDO. All 48 manuscripts submitted to this SI were subject to the same rigurious review process assessing their overall quality and significance. A total of 20 papers were accepted for publication in this special issue.

The rest of this guest editorial article is organized as follows. Section 2 provides a brief review of the state of the art in four AMDO subtopics, namely, human motion analysis, human pose estimation, deformable segmentation, and

${ }_{20}$ face analysis. Section 3 summarises the papers accepted for the Special Issue. We conclude with a brief outlook to the future in Sec. 4 .

\section{Articulated Motion and Deformable Objects}

We structure the review of the AMDO literature into four main topics, namely, human motion analysis, human pose estimation, deformable shape segmentation, and facial analysis. For each subtopic, we review the commonly used benchmark datasets and the main state-of-the-art methods published in the last two years. The papers published in this special issue are referred to by their unique number, e.g. $S I_{1}, S I_{2}$.

\subsection{Human Motion Analysis}

The two main topics of human motion analysis are action and gesture recognition. Recently, the use of multimodal data in the context of human motion analysis has received a lot of attention in the literature. RGB, depth, and skeletal information are the commonly considered modalities for multimodal action 
Table 1: Statistics of popular benchmark datasets for human motion analysis in the field of AMDO. All datasets are used at least once by accepted manuscripts in this special issue.

\begin{tabular}{c|c|c|c|c|c|c}
\hline index & Dataset & Year & Modality & \#Class & \#Subjects & \#Samples \\
\hline 1 & $\begin{array}{c}\text { MSR Action3D, } \\
{[1]}\end{array}$ & 2010 & $\begin{array}{c}\text { RGB } \\
\text { skeleton }\end{array}$ & 20 & 10 & 567 \\
\hline 2 & $\begin{array}{c}\text { MSRC-12, } \\
{[2]}\end{array}$ & 2012 & skeleton & 12 & 30 & 594 \\
\hline 3 & $\begin{array}{c}\text { MSR DailyActivity3D, } \\
{[3]}\end{array}$ & 2012 & $\begin{array}{c}\text { RGB-D } \\
\text { skeleton }\end{array}$ & 16 & 10 & 320 \\
\hline 4 & $\begin{array}{c}\text { UTKinect, } \\
{[4]}\end{array}$ & 2012 & $\begin{array}{c}\text { RGB-D } \\
\text { skeleton }\end{array}$ & 10 & 10 & 200 \\
\hline 5 & $\begin{array}{c}\text { SBU Kinect, } \\
\text { Interaction [5] }\end{array}$ & 2012 & $\begin{array}{c}\text { RGB-D } \\
\text { skeleton }\end{array}$ & 7 & 8 & 300 \\
\hline 6 & $\begin{array}{c}\text { NTU RGB+D, } \\
{[6]}\end{array}$ & 2016 & $\begin{array}{c}\text { RGB-D } \\
\text { skeleton }\end{array}$ & 60 & 40 & 56880 \\
\hline 7 & $\begin{array}{c}\text { DHG-14/28, } \\
{[7]}\end{array}$ & 2016 & $\begin{array}{c}\text { RGB-D } \\
\text { skeleton }\end{array}$ & 14 & 20 & 2800 \\
\hline 8 & $\begin{array}{c}\text { Montalbano V2, } \\
{[8]}\end{array}$ & 2014 & $\begin{array}{c}\text { Depth } \\
\text { skeleton }\end{array}$ & 20 & 27 & 13858 \\
\hline 9 & $\begin{array}{c}\text { MIVIA action, } \\
{[9]}\end{array}$ & 2014 & RGB-D & 7 & 14 & 500 \\
\hline 10 & $\begin{array}{c}\text { NATOPS gesture, } \\
{[10]}\end{array}$ & 2011 & RGB-D & 24 & 20 & 9600 \\
\hline
\end{tabular}

and gesture recognition. In this section, first, we provide a brief introduction to action and gesture recognition datasets. Then we review the state-of-the-art methods in this topic area.

\subsubsection{Benchmark Datasets for action and gesture recognition}

Table 1 shows the commonly used datasets for isolated or continuous action and gesture recognition, and summarises the key statistical attributes of these datasets, namely data modality, number of classes, number of subjects, and number of samples. Each dataset is used at least once in the manuscripts accepted for this special issue.

MSR Action3D [1]. It includes 567 sequences, including twenty actions (namely, high arm waving, horizontal arm waving, hammering, hand catching,

45 forward punching, high throwing, drawing $\mathrm{x}$, drawing a tick, drawing a circle, hand clapping, two hand waving, side-boxing, bending, forward kicking, side kicking, jogging, tennis serve, golf swing, pickup and throw), each action being 
performed three times by ten subjects. The data is recorded from a fixed point of view while the subjects are facing the camera.

$M S R C$-12 [2]. It comprises thirty subjects performing twelve gestures. These gestures are grouped into two categories: iconic and metaphoric gestures. The iconic gestures directly correspond to real world actions and represent first people shooter (FPS) gaming actions. It contains six FPS gaming actions: crouching, shooting, throwing, using night goggles, changing weapon and kicking.

MSR DailyActivity3D [3]. This dataset contains sixteen daily human activities in a living room: drinking, eating, reading a book, calling a cellphone, writing on a paper, using a laptop, using a vacuum cleaner, cheering up, sitting still, tossing paper, playing a game, laying down on a sofa, walking, playing a guitar, standing up, sitting down. Ten subjects are recorded performing these actions while sitting on the sofa or standing close to the sofa. The camera is fixed in front of the sofa. The dataset also provides depth and skeleton data.

UTKinect [4]. Ten types of human action are recorded twice by ten subjects. The actions include walking, sitting down, standing up, picking up, carrying, throwing, pushing, pulling, waving, clapping hands. The actions were recorded from a variety of views. The dataset is composed of 200 sequences, recording RGB-D data and skeleton joint locations.

SBU Kinect Interaction [5]. It has eight classes which are commonly used in two-person interactions, namely, approaching, departing, pushing, kicking, punching, exchanging objects, hugging, and shaking hands. This dataset is challenging because of the similarity of some actions in terms of motion (e.g., exchanging object and shaking hands). RGB and depth video with 15 frames per second are provided, with an image resolution of $640 \times 480$.

$N T U R G B+D[6]$. It is a large scale dataset for human action recognition, which consists of 56,880 action samples with four different data modalities for 75 each sample: RGB videos, depth map sequences, 3D skeletal data, and infrared videos. It has 60 classes in total, which are divided into three major groups: 40 daily actions (i.e., drinking, eating, etc.), 9 health-related action (i.e., sneezing, staggering, etc.), and 11 interactions (i.e., punching, kicking, etc.). 
DHG-14/28 [7]. The dynamic hand gesture dataset DHG-14/28 has fourteen

\subsubsection{The state-of-the-art in Human motion analysis}

Table 2 shows a comparison of different state-of-the-art methods on the ten datasets used by the papers on human motion analysis accepted for the SI. Three main tasks are considered, namely, action detection, isolated action/gesture recognition, and continuous gesture recognition. For different tasks, the evalution, F1-score for action detection and Jaccard index (JI) for continuous gesture recognition. In Table 2, we show the performance of state-of-the-art methods in different datasets and provide the details of the specific evaluation protocols 
Table 2: A Comparison of state-of-the-art methods on the benchmark action/gesture datasets listed in Table 1. All cited papers have been published in the last two years. Protocol A: half of the subjects are used for training (i.e. odd subjects) and the rest for testing (i.e. even subjects); Protocol B: average over all splits; Protocol C: leave-one-subject-out; Protocol D: training with the first 5 subjects, testing with other 10 subjects; acc: accuracy; JI: Jaccard Index.

\begin{tabular}{|c|c|c|c|c|}
\hline Dataset & Method & \multicolumn{2}{|c|}{ Metrics } & Protocol and notes \\
\hline \multirow{8}{*}{ MSR Action3D [1] } & $\mathrm{CNN}+\mathrm{LSTM}\left[S I_{1}\right]$ & $\operatorname{acc}(\%)$ & $95.7 / 96.0$ & $\mathrm{~A} / \mathrm{B}$ \\
\hline & $\operatorname{DSRF}\left[S I_{3}\right]$ & $\operatorname{acc}(\%)$ & 95.24 & no specific mention \\
\hline & Bag of Gesturelets $\left[S I_{8}\right]$ & $\operatorname{acc}(\%)$ & 96.49 & A \\
\hline & Pose and Kinematics $\left[S I_{19}\right]$ & $\operatorname{acc}(\%)$ & 96.77 & A \\
\hline & MIMTL[11], 2017 & $\operatorname{acc}(\%)$ & 96.37 & A \\
\hline & trajectorylet+exemplar-SVMs[12], 2017 & $\operatorname{acc}(\%)$ & 97.9 & A \\
\hline & $\operatorname{DMMs}[13], 2016$ & $\operatorname{acc}(\%)$ & 90.5 & A \\
\hline & Riemannian Manifold[14], 2016 & $\operatorname{acc}(\%)$ & 96.97 & A \\
\hline \multirow{10}{*}{ MSRC-12 [2] } & $\operatorname{DSRF}\left[S I_{3}\right]$ & $\operatorname{acc}(\%)$ & $95.64 / 95.36$ & $\mathrm{C} / \mathrm{A}$ \\
\hline & Bag of Gesturelets $\left[S I_{8}\right]$ & F1-score & 89.8 & $\mathrm{C}$ \\
\hline & Clustered Spatiotemporal Manifolds $\left[S I_{9}\right]$ & F1-score & 77.3 & $\mathrm{C}$ \\
\hline & Enhanced Skeleton $\left[S I_{11}\right]$ & $\operatorname{acc}(\%)$ & 96.62 & A \\
\hline & Pose Lexicon $\left[S I_{18}\right]$ & $\operatorname{acc}(\%)$ & 92.03 & - \\
\hline & Pose and Kinematics $\left[S I_{19}\right]$ & $\operatorname{acc}(\%)$ & 91.20 & $\mathrm{C}$ \\
\hline & View Invariant Information+CNNs[15], 2017 & $\operatorname{acc}(\%)$ & 96.62 & A \\
\hline & Trajectorylet+Exemplar-SVMs[12], 2017 & $\operatorname{acc}(\%)$ & $94.9 / 95.1$ & $\mathrm{~A} / \mathrm{C}$ \\
\hline & Encoded Spatial-temporal Information $+\mathrm{CNN}[16], 2016$ & $\operatorname{acc}(\%)$ & 94.27 & A \\
\hline & Joint Trajectory Maps+CNNs[17], 2016 & $\operatorname{acc}(\%)$ & 93.12 & A \\
\hline \multirow{4}{*}{ MSR DailyActivity3D [3] } & $\mathrm{CNN}+\mathrm{LSTM}\left[S I_{1}\right]$ & $\operatorname{acc}(\%)$ & 63.1 & no specific mention \\
\hline & DSSCA SSLM[18], 2017 & $\operatorname{acc}(\%)$ & 97.5 & A \\
\hline & Unsupervised training[19], 2017 & $\operatorname{acc}(\%)$ & 86.9 & no specific mention \\
\hline & MFSK+BOW [20], 2016 & $\operatorname{acc}(\%)$ & 95.7 & $\mathrm{C}$ \\
\hline \multirow{6}{*}{ UTKinect [4] } & $\mathrm{CNN}+\mathrm{LSTM}\left[S I_{1}\right]$ & $\operatorname{acc}(\%)$ & 99.0 & $\mathrm{C}$ \\
\hline & $\operatorname{DSRF}\left[S I_{3}\right]$ & $\operatorname{acc}(\%)$ & 97.85 & A \\
\hline & Geometric Feature+LSTM[21], 2017 & $\operatorname{acc}(\%)$ & 95.96 & A \\
\hline & $\mathrm{VLDA}+\mathrm{LMNN}+\mathrm{k}-\mathrm{NN}[22], 2017$ & $\operatorname{acc}(\%)$ & 98 & C \\
\hline & JSG (top-K RVJRDs)+JSGK[23], 2017 & $\operatorname{acc}(\%)$ & 98.3 & $\mathrm{C}$ \\
\hline & Triplet motion + LBP[24], 2016 & $\operatorname{acc}(\%)$ & 98.0 & 3-fold cross-validation \\
\hline \multirow{4}{*}{ Kinect Interaction $[5]$} & Motion Information+CNN $\left[S I_{5}\right]$ & $\operatorname{acc}(\%)$ & 90.98 & 5-fold cross validation \\
\hline & Geometric Feature+LSTM[21], 2017 & $\operatorname{acc}(\%)$ & 99.02 & 5-fold cross validation \\
\hline & SkeletonNet [25], 2017 & $\operatorname{acc}(\%)$ & 93.47 & 5-fold cross validation \\
\hline & Co-occurence feature+LSTM [26], 2016 & $\operatorname{acc}(\%)$ & 90.41 & 5-fold cross validation \\
\hline \multirow{5}{*}{ NTU RGB+D [6] } & $\mathrm{CNN}+\mathrm{LSTM}\left[S I_{1}\right]$ & $\operatorname{acc}(\%)$ & $67.5 / 76.21$ & cross-subject/cross-view \\
\hline & Enhanced Skeleton $\left[S I_{11}\right]$ & $\operatorname{acc}(\%)$ & $80.03 / 87.21$ & cross-subject/cross-view \\
\hline & DSSCA SSLM[18], 2017 & $\operatorname{acc}(\%)$ & 74.9 & cross-subject \\
\hline & Joint distance maps+CNN [27], 2017 & $\operatorname{acc}(\%)$ & $76.2 / 82.3$ & cross-subject/cross-view \\
\hline & Part-aware LSTM Network [6], 2016 & $\operatorname{acc}(\%)$ & $62.03 / 70.27$ & cross-subject/cross-view \\
\hline \multirow{2}{*}{ DHG-14/28 [7] } & $\mathrm{CNN}+\mathrm{LSTM}\left[S I_{1}\right]$ & $\operatorname{acc}(\%)$ & $85.6 / 81.1$ & $\mathrm{C}$; acc of $14 / 28$ gestures \\
\hline & Geometric shape+fisher vector+SVM[7], 2016 & $\operatorname{acc}(\%)$ & $83.07 / 79.14$ & $\mathrm{C}$; acc of $14 / 28$ gestures \\
\hline \multirow{2}{*}{ Montalbano V2 [8] } & $\mathrm{CNN}+\mathrm{LSTM}\left[S I_{1}\right]$ & JI & 79.15 & no specific mention; \\
\hline & Moddrop [28], 2016 & JI & 83 & no specific mention; \\
\hline \multirow{3}{*}{ MIVIA action [9] } & Motion Information+CNN $\left[S I_{5}\right]$ & $\operatorname{acc}(\%)$ & 93.37 & $\mathrm{C}$ \\
\hline & Key poses $+\mathrm{CNN}[29], 2017$ & $\operatorname{acc}(\%)$ & 93.37 & $\mathrm{C}$ \\
\hline & String kernel framework[30], 2016 & $\operatorname{acc}(\%)$ & 95.4 & $\mathrm{C}$ \\
\hline \multirow{2}{*}{ NATOPS gesture [10] } & Motion Information+CNN $\left[S I_{5}\right]$ & $\operatorname{acc}(\%)$ & $72.58 / 86.58$ & D; top-1/top-2 accuracy \\
\hline & Random forest[31], 2017 & $\operatorname{acc}(\%)$ & 88.1 & no specific mention \\
\hline
\end{tabular}


for each cited paper.

The listed methods in Table 2 can be grouped into two categories. The first category includes traditional methods for motion analysis, which consist of a pipeline commencing with feature extraction, through feature encoding, to classification. In order to extrtact efficient features from image sequences/videos, some heuristically designed descriptors are proposed, such as the Dual SquareRoot Function (DSRF) descriptor in $S I_{3}$, Gesturelets in $S I_{8}$, to complement trajectorylet [12], mixed features around sparse keypoints (MFSK) [20], cooccurence feature [26], triplet motion and local binary pattern (LBP) [24]. For feature encoding, the representative methods include bag of visual words (BoVW) [20], vector of locally aggregated descriptors (VLAD) [22] and fisher 120 vector $(\mathrm{FV})[7]$. For the decision-making stage, the popular classifiers applied to the datasets in the Table 2 include KNN [20, 22], SVM [12, 7] and random forest [31].

The second category comprises the deep learning based methods. It consists of Convolutional neural networks (CNNs), Long Short Term Memory networks 125 (LSTM) and mixed architecture based approaches. CNN-based methods typically encode image sequences or skeletons as dynamic images that capture the spatio-temporal or skeleton-based motion information [15, 16, 17, 27], and then apply CNN for image-based recognition. LSTM-based methods extract geometric or co-occurence features $[21,26]$ from each frame and train a model which encodes the spatio-temporal information content. Some approaches combine CNN and LSTM to realise an action recognition capability. For example, $S I_{1}$ combines CNN and LSTM in a two-stage training strategy designed to optimise the parameters of a CNN+LSTM framework.

For human motion analysis, there are many other CNN-based methods which 135 are not mentioned in this section. For a more detailed survey the reader is referred to $[32,33,34,35,36]$. 
Table 3: Statistics of the HumanEva dataset used for benchmarking human pose estimation algorithms.

\begin{tabular}{c|c|c|c|c|c|c}
\hline Index & Dataset & Year & Modality & \#Class & \#Subjects & \#Samples \\
\hline 1 & HumanEva [37] & 2010 & RGB,skeleton & 6 & 4 & 40000 \\
\hline
\end{tabular}

\subsection{Human Pose Estimation}

\subsubsection{Benchmark Datasets for Human Pose Estimation}

Table 3 summarises the attributes of the human pose estimation dataset used for evaluation by some of the work presented in this special issue.

HumanEva [37]. This dataset contains six classes and about 40K samples recorded by four subjects. It comprises synchronized images and motion capture data and is a standard benchmark for 3D human pose estimation. The output pose is a vector of $153 \mathrm{D}$ joint coordinates.

\subsubsection{The state-of-the-art in Human pose estimation}

Table 4 shows some methods that use the HumanEva dataset. All the listed papers have been published within the last two years. In $S I_{4}$, the authors build virtual humans via a professional free and open-source 3D computer graphics software called Blender ${ }^{1}$ and a free software enabling the creation of realistic $3 \mathrm{~d}$ human makehuman ${ }^{2}$ data. These avatars can be animated to simulate realistic actions based on the motion capture data. One of the main advantages is that one can automatically generate ground truth data. The software thus saves a lot of effort by avoiding manual data collection and annotation. However, as the authors said "due to either the lack of motion capture file for importation into the graphics software or the lack of 3D ground truth, this makes a quantitative evaluation and comparison on public datasets difficult". Therefore, only qualitative results on the HumanEva dataset are presented in $S I_{4}$.

Table 4 lists the recently published papers based on deep learning. Federica

\footnotetext{
${ }^{1}$ https://www.blender.org/

${ }^{2}$ http://www.makehuman.org/
} 
et al.[38] present a SMPLify framework that falls within the classical paradigm of bottom up estimation followed by top down verification (generative model). For the bottom-up estimation, a CNN-based method called DeepCut is used to predict the $2 \mathrm{D}$ body joint locations. The role of the top-down strategy is to fit the body shape to the 2D joints via SMPL [39]. The objective function penalizes the error between the projected 3D model joints and detected 2D joints.

Zhou et al.[40] propose a sparseness meets deepness (SMP) algorithm to address the challenge of 3D full-body human pose estimation from a monocular image sequence. It consists of a novel synthesis of a deep learning-based 2D part regressor, a sparsity driven 3D reconstruction approach and a 3D temporal smoothness prior. This joint consideration combines the discriminative power of the state-of-the-art 2D part detectors, the expressiveness of 3D pose models and regularization by way of aggregating information over time.

Bugra et al.[41] propose a trainable fusion scheme to fuse 2D and 3D image cues for monocular body pose estimation. It consists of two streams. The first CNN stream is used to predict the $2 \mathrm{D}$ joint locations and the corresponding uncertainties. The second one leverages all 3D image cues by processes applied directly to the input image. The outputs of these two streams are then fused to obtain the final 3D human pose estimation.

The work in [42] presents a robust marker-less human motion capture algorithm that can track articulated joint motion in challenging indoor and outdoor scenes. It combines the strengths of a discriminative image-based joint detection method with a model-based generative motion tracking algorithm through a unified pose optimization energy. The discriminative part-based pose detection method is implemented using CNNs. It estimates unary potentials for each joint of a kinematic skeleton model. These unary potentials serve as the basis of a probabilistic extraction of pose constraints for tracking by using a weighted sampling from a pose posterior that is guided by the model. In the final energy formula, it combines these constraints with an appearance-based model-to-image similarity term. 
Table 4: A comparison of the state-of-the-art human pose estimation methods evaluated on the HumanEva [37] dataset. The protocol used to obtain the results in this table involves sequences $\mathrm{S} 1, \mathrm{~S} 2, \mathrm{~S} 3$, for training and the validation sequences of all subjects for testing. The evaluation metric is the average joint error in centimeter $(\mathrm{cm})$.

\begin{tabular}{cccc}
\hline \multirow{2}{*}{ Method } & \multicolumn{3}{c}{ Metrics:jont error $(\mathrm{cm})$} \\
& walking & jogging & boxing \\
\hline Invariants moments [SI_4] & \multicolumn{2}{c}{ only qualitative results provided } \\
Marker-less Motion Capture [42], 2017 & 6.65 & - & 6.00 \\
Trainable Fusion [41], 2017 & 2.44 & - & - \\
SMP [40], 2016 & 3.81 & 3.68 & - \\
SMPLify [38], 2016 & 7.72 & - & 8.28 \\
\hline
\end{tabular}

\subsection{Deformable Shape Segmentation}

190

\subsubsection{Benchmark Datasets for Deformable Shape Segmentation}

Princeton Segmentation Benchmark (PSB) Dataset [43]. This dataset has been intensively used to evaluate 3D shape segmentation and 3D shape retrieval algorithms. It has 19 different object categories with 20 objects for each category, which results in a total of 380 models (see Table 5).

In order to evaluate segmentation methods, some popular metrics are used including rand index, cut discrepancy, hamming distance and consistency error. Rand index measures the similarity between two segmentations of the same shape. From a mathematical point of view, rand Index is related to the accuracy, but is applicable even when class labels are not used. Rand index error is equal to one minus the Rand Index. Cut discrepancy is a boundary-based method evaluating the distance between different cuts. It sums the distances from points along the cuts in the computed segmentation to the closest cuts in the ground truth segmentation, and vice-versa. Hamming Distance is a regionbased method which measures the number of substitutions required to change the assignment of one region into another. Hamming Distance is directional, hence it includes underdetection rate $(\mathrm{Rm})$ and false alarm (Rf) distances. Con- 
Table 5: Statistics of the key attributes of the popular benchmarking dataset for deformable shape segmentation in the field of AMDO. The dataset is used at least once by the manuscripts accepted for this special issue.

\begin{tabular}{c|c|c|c|c}
\hline Dataset & Year & \#Classes & \#Objects & \#Samples \\
\hline PSB [43] & 2004 & 19 & 20 & 380 \\
\hline
\end{tabular}

Table 6: A comparison of the state-of-the-art methods on the PSB dataset [43]. The metrics include (refer to [44] for more details): Cut Discrepancy (CD), Hamming Distance (HD), Rand Index (RI) and Consistency Error (CE), Global Consistency Error (GCE), Local Consistency Error (LCE). Hamming Distance is directional, hence it includes underdetection rate (Rm) and false alarm (Rf) distances.

\begin{tabular}{c|c|c|c|c|c|c}
\hline & \multicolumn{6}{|c}{ Metrics } \\
\hline Paper & 0.149 & 0.090 & 0.118 & 0.124 & \multicolumn{2}{c}{0.065} \\
\hline \multirow{2}{*}{ Multi-view RNN [45], 2017 } & CD & HD & Hamming-Run & Hamming-Rf & GCE & LCE \\
\cline { 2 - 7 } & 0.144 & 0.075 & 0.061 & 0.089 & 0.060 & 0.041 \\
\hline Stacked auto-encoders [46], 2016 & \multicolumn{6}{|c}{ RI: 0.118 } \\
\hline
\end{tabular}

sistency Errors, whether the global version (GCE) or local version (LCE), are used to compute the hierarchical differences and similarities between segmentations. They are based on the theory that the organisation of perceptual information by humans imposes a hierarchical tree structure on perceived objects. For all four metrics, a smaller value indicates a better result. These metrics are shown in Table 6.

\subsubsection{State-of-the-art on Deformable Shape Segmentation}

Table 6 shows a comparison of different methods on the PSB dataset [43]. Truc et al. [45] present a multi-view RNN (MV-RNN) algorithm for 3D mesh segmentation. It combines CNNs and a two-layer LSTM to yield coherent segmentation of 3D shapes. The image-based CNN effectively generates the edge probability feature map while the LSTM correlates the edge maps across different views and outputs a well-defined per-view edge image. From Table 6, one can see that the performance of $S I_{7}$ is comparable to that of MV-RNN for 
Table 7: The statistics of key attributes of popular benchmarking datasets for face analysis in the field of AMDO. All datasets are used at least once by accepted manuscripts in this special issue.

\begin{tabular}{cccccc}
\hline Dataset & Images & Subjects & Age groups & Gender & In the wild \\
Adience [51] & 26,580 & 2,284 & 8 & Yes & Yes \\
IoG [52] & 5,080 & 28,231 & 7 & Yes & Yes \\
MORPH II [53] & 55,134 & 13,000 & Accurate ages & Yes & No \\
\hline
\end{tabular}

different evaluation metrics.

The work [46] proposes an unsupervised method for 3D shape segmentation. After over-segmenting the shapes into primitive shapes, it generates high-level features from low-level features of each patch by using stacked auto-encoders. High-level features are then used for segmenting a single shape or co-segmenting a group of shapes.

\subsection{Face Analysis}

In the area of computer vision and patter recognition, face analysis [47, 48, $49,50]$ is a popular and hot research direction. However, in this section, we limit the review techniques and datasets used in the papers contained in the special issue.

\subsubsection{Benchmarking Datasets for Face Analysis}

There are three popular benchmarking datasets related to age and gender analysis: Adience [51], IoG [52] and MORPH II [53]. Some key statistics of these three datasets are listed in Table 7.

The face images of Adience and IoG datasets are collected in the wild. Both datasets contain age group and gender information. For MORPH II dataset, 50 thousand images have been collected in a controlled environment. Different from Adience and IoG datasets, MORPH II dataset provides accurate age information 240 for each face image. 
Table 8: The key attribute statistics of popular benchmarking datasets used for age, gender and ethnicity analysis in the field of AMDO.

\begin{tabular}{c|ccc|ccc|cc}
\hline \multirow{2}{*}{ Methods } & \multicolumn{3}{|c|}{ Adience [51] } & \multicolumn{3}{c|}{ IoG [52] } & \multicolumn{2}{c}{ Morph II [53] } \\
& Age & Gender & Protocol & Age & Gender & Protocol & Age & Protocol \\
\hline Deep Attention [SI $\left.I_{10}\right]$ & 0.6108 & 0.9300 & - & 0.6 & 0.8690 & - & 2.56 & $80-20^{4}$ \\
AgeED ${ }^{1}[54], 2017$ & - & - & - & - & - & - & 2.52 & $80-20$ \\
MRNPE [55], 2017 & - & - & - & - & - & - & 2.73 & CBSR $^{5}$ \\
$\operatorname{DEX}^{2}[56], 2016$ & 0.64 & - & - & - & - & - & 2.68 & - \\
Soft softmax [57], 2016 & - & - & - & - & - & - & 3.03 & CBSR \\
Cascaded CNN [58], 2016 & 0.5288 & - & FF-SECV $^{3}$ & - & - & - & - & - \\
RoR[59], 2017 & - & 0.9059 & FF-SECV $^{3}$ & - & 0.9073 & FF-SECV $^{3}$ & - & - \\
OR-CNN[60], 2016, & - & - & - & - & - & - & 3.27 & - \\
\hline
\end{tabular}

[1] AgeED: Age Encoding + Decoding; [2] DEX: Deep EXpectation;[3] FF-SECV: five-fold, subject-exclusive cross-validation protocol; [4] 80-20: $80 \%$ for training and $20 \%$ for testing; [5] http://www.cbsr.ia.ac.cn/users/dyi/agr.html;

\subsubsection{The state-of-the-art in Face analysis}

There are many subtopics of face analysis, such as face verification and recognition [61, 62], facial expression recognition [63], and face attribute analysis [57] (i.e. age estimation, gender and ethnicity recognition), to mention just a few. Some solutions already achieve very promising performance that in many respects exceeds that of human face perception $[61,62]$. It is out of the scope of this editorial to provide a comprehensive coverage of the recent advances in the field of face analysis. We only focus on face attribute analysis, such as age estimation, ethnicity and gender recognition. Some recently published methods that were evaluated on the above three datasets are listed in Table 8 . Unfortunately, there is a lack of standardisation and different publications often use different protocols for evaluation. For example, on MORPH II dataset, MRNPE [55] and Soft softmax [57] evaluate their models with CBSR protocol while $S I_{10}$ and AgeED [54] use 80-20 protocol.

Tan et al. [54] propose a group-based method for accurate age estimation. First, they propose an age group-n encoding (AGEn) method, where adjacent ages are merged into the same category. Note that ages merged into the same group would be regarded as independent classes in the training stage. On this 
basis, authors transform the age estimation problem into a series of binary classification sub-problems. Subsequently, deep CNNs realising multiple classifiers are trained for age group classification. For testing, an age decoding stage is proposed to deduce the estimated age from the age group classification result. As shown in Table 8, this method achieves a MAE of 2.52 with the 80-20 protocol.

The work in [57] proposes a soft softmax loss function for age estimation, where each face image is labeled with a Gaussian label distribution rather than a single label value in softmax loss function. Compared with the traditional definitions, the proposed soft softmax loss function considers not only the chronological age but also its adjacent ages. The authors show the effectiveness of their proposal for age estimation achieving a MAE of 3.03 with a shallow network 270 (AlexNet) on the MORPH II dataset.

Chen et al. [55] propose a Multi-Region Network Prediction Ensemble (MRNPE) for high-accuracy age estimation by leveraging both global and local context information. The model includes multiple sub-networks, where each sub-network takes both a global face image and a local region as input, e.g., face $275+$ eye, face + mouth and face + nose. Then, the average over the predictions of all sub-networks is reported as the final predicted age. One disadvantage of this work is that it needs an ensemble of networks to achieve high performance.

Rothe et al. [56] propose a DEX (Deep EXpectation) framework for real and apparent age estimation. They regard age estimation as a deep classification problem followed by a softmax expected value refinement. DEX is a very popular method for age estimation. It won the first place in the Chalearn LAP challenge 2015. In this paper, Rothe et al. also introduce the largest public dataset of face images, IMDB-WIKI datatset, which contains age and gender information for each face image. One drawback of this dataset is that its labels are noisy, as a result of being calculated based on the date of birth of the corresponding celebrity and the year when the photo was taken. Thus, this dataset is usually used for pretraining rather than evaluation.

The work in [58] presents a coarse-to-fine framework for age estimation in unconstrained environment. First, age group classification is carried out to 

issue. The papers are grouped in the above mentioned four AMDO subtopoics (14 papers on human motion analysis, three papers on pose estimation, one on deformable shape, and two on face analysis).

\subsection{Human Motion Analysis}

$S I_{1}$ : The paper "Convolutional Neural Networks and Long Short-Term Memory for Skeleton-Based Human Activity and Hand Gesture Recognition" by Juan C. Núñez, Raúl Cabido, Juan J. Pantrigo, Antonio S. Montemayor and 
José F. Vélez, proposes a deep learning-based method for skeleton-based human activity and hand gesture recognition. It combines CNN and Long Short-Term Memory (LSTM) recurrent networks. A two-stage training strategy is applied to update $\mathrm{CNN}+\mathrm{LSTM}$ framework parameters. An exhaustive experimental evaluation on publicly available data benchmarks (i.e. MSR Action3D, MSR DailyActivity3D, UTKinect, NTU RGB+D, DHG-14/28, and Montalbano V2) is presented, showing the proposed method to be competitive in relation to CNN+LSTM architecture for activity recognition in video sequences, but only using skeleton and achieving competitive results on five datasets.

$S I_{2}$ : The paper "Hand Action Detection from Ego-centric Depth Sequences with Error-correcting Hough Transform" by Chi Xu, Lakshmi N Govindara3зо jan and Li Cheng, presents an effective and efficient solution for hand action detection from mobile ego-centric depth sequences. It proposes a novel errorcorrecting mechanism to tackle the issue of incorrect votes generated by the Hough transform. The authors also provide a comprehensive in-house annotated ego-centric hand action dataset. We believe this will open new research directions in ego-centric hand action detection. The proposed method delivers favorable performance in real time (about 112 frame/second) on their proposed real-life dataset. It is related to the work in [66], which uses the concept "snippets" for action recognition, but applied to Ego-centric hand detection. Moreover, the released real-life dataset of this paper is also likely push the state of the art in Ego-centric hand detection research.

$\mathrm{SI}_{3}$ : The paper "A Flexible Trajectory Descriptor for Articulated Human Action Recognition" by Yao Guo, Youfu Li and Zhanpeng Shao, proposes an articulated skeleton representation by modeling the skeleton information as interconnections of multiple rigid bodies for action recognition. In this method, 345 six-dimensional rigid body motion trajectories are represented by the invariant Dual Square-Root Function (DSRF) descriptor. The concept of Virtual Rigid Body (VRB) configuration is introduced to produce compact mid-level features for representing the movement of each body part. The Most Informative Part 
(MIP) trajectory is then used to select a subset of consistency and activity body Background Subtraction" by Mohammadreza Babaee, Duc Tung Dinh and Gerhard Rigoll, proposes a deep CNN architecture (namely DeepBS) for background subtraction from video sequences. The input frame along with the corresponding background image are patch-wise processed. During training, the hypotheisised dividual patches into a single output frame, the output frame is post-processed, yielding the final output segmentation. The proposed method is evaluated on different data-sets, and shown to outperform the existing algorithms as mea- 
in CDnet 2014. It is similar to the CNN-based work of [68] which uses a fixed background model. However [68] is defined for a concrete video scenario and will require re-training for other scenes with scene-specific data, while $S I_{6}$ can handle various video scenes.

385 ation based on Motion Capture Data" by Fotini Patrona, Anargyros Chatzitofis, Dimitrios Zarpalas and Petros Daras, presents a motion analysis framework for real-time action detection, recognition and evaluation of motion capture data based on the pose and kinematics information. First, automatically computed tion involvement. Then the bag of gesturelets (BoG) model is employed for data representation of each sample and kinetic energy based descriptor sampling is performed before a codebook construction. The automatically segmented and recognized action samples are subsequently fed into a framework evaluation can effectively be used for unsupervised gesture/action training. This work is similar to bag of visual words model $[69,20]$ widely used in video-based recognition, but here being specifically designed for a motion analysis task.

$S I_{9}$ : The paper "Linear Latent Low Dimensional Space for Online Early 400 Action Recognition and Prediction" by Victoria Bloom, Vasileios Argyriou, and Dimitrios Makris, utilizes joint motion data for recognizing actions in linear latent spaces. It operates online and in real time. It is based on supervised learning and dimensionality reduction techniques, which derive a representation for high dimensional nonlinear actions in a linear laten low dimensional space. The proposed method is evaluated on well-know datasets. Compared to the state of the art methods, the proposed approach exhibits high accuracy and low latency properties. Previous works $[70,71,72]$ considered early action recognition, online action recognition and action prediction as independent events, while $S I_{9}$ tackles these three tasks jointly with the proposed Clustered 410 Spatio-Temporal Manifolds. 
$S I_{11}$ : The paper "Enhanced skeleton visualization for view invariant human action recognition" by Mengyuan Liu, Hong Liu, and Chen Chen, proposes a new enhanced skeleton visualization method for action recognition. The authors develop a sequence-based view invariant transform, based on spatio-temporal (FFW-CRBMs) [74]. However, FFW-CRBMs require three-dimensional labeled information for accurate predictions which is not typically available.

$S I_{14}$ : The paper "Spatio-Temporal Union of Subspaces for Multi-body Nonrigid Structure-from-Motion" by Suryansh Kumar, Yuchao Dai, and Hongdong

${ }_{440} \mathrm{Li}$, proposes a unified framework to jointly segment and reconstruct multiple non-rigid objects. It exploits the structure of the scene along the temporal and 
spatial directions, modelled in terms of 3D non-rigid deformations. The spatiotemporal representation not only provides competitive 3D reconstruction but also outputs a robust segmentation of multiple non-rigid objects. The resultant optimization problem is solved using the Alternating Direction Method of Multipliers (ADMM). The experimental results show the superiority of the method, compared to the state-of-the-art. Compared with other similar methods [75, 76], the proposed method of $S I_{14}$ can learn the affinity matrices to exploit efficient spatio-temporal clustering structures.

$S I_{16}$ : The paper "Adaptive Compressive Tracking based on Locality Sensitive Histograms" by Sixian Chan, Xiaolong Zhou, Junwei Li, and Shenyong Chen, proposes an adaptive compressive tracking algorithm which is locality sensitive, and thus robust to illumination variations. A new update mechanism is used to preserve stable features while avoiding noisy appearance variations during tracking. Furthermore it includes a trajectory rectification method to refine the tracking accuracy. The experimental results conducted on a benchmarking dataset show that the tracker achieves the state-of-the-art performance. It is related to the works $[77,78]$ on compressive tracking with color information. Compared to $[77,78], S I_{16}$ presents an updating mechanism to preserve stable features.

$S I_{18}$ : The paper "Semantic Action Recognition by Learning a Pose Lexicon" by Lijuan Zhou, Wanqing Li, Philip Ogunbona, and Zhengyou Zhang, proposes a semantic representation, exploiting a pose lexicon, for action recognition. Each action is represented by a sequence of semantic poses extracted from an associated textual instruction. A visual pose model, defined as a Gaussian mixture, is learned from training samples to characterize the likelihood of an observed visual frame being generated by a visual pose. A pose lexicon model is learned using an extended Hidden Markov Model (HMM) to encode the probabilistic mapping between hidden visual poses and semantic poses sequences. With the lexicon, action classification is formulated as a problem of finding the sequence of semantic poses that best fits the sequence of visual frames as measured in terms of posterior probability. The efficacy of the proposed method is evaluated 
on different datasets (i.e. MSRC-12, WorkoutUOW-18, and Combined-17 action datasets) using cross-subject, cross-dataset and zero- shot protocols. $S I_{18}$ is an extension of the work in [79]. Compared with [79], $S I_{18}$ jointly generates visual pose sequences and aligns them to semantic pose sequences.

$S I_{19}$ : The paper "Motion Analysis: Action Detection, Recognition and Evaluation based on motion capture data" by Fotini Patrona, Anargyros Chatzitofis, Dimitrios Zarpalas, and Petros Daras, proposes a new framework for real-time action detection and recognition. Automatic and dynamic weighting, altering the joint data significance based on the involved action, and Kinetic energybased descriptor sampling, are employed for efficient action segmentation and labeling. The automatically segmented and recognized action instances are subsequently fed to the action evaluation stage of the framework. It compares them with the reference instances, estimating their similarity. The experimental results obtained on MSR-Action3D and MSRC12 datasets, provide evidence that the proposed method outperforms state-of-the-art methods by $0.5-6 \%$ in all datasets. $S I_{19}$ is similar to the work in [80]. Compared with [80], automatic feature weighting at the frame level is employed in $S I_{19}$ which also uses all 20 joints.

$S I_{20}$ : The paper "Active garment recognition and target grasping point detection using deep learning" by Enric Corona, Guillem Alenya, Antoni Gabas, and Carme Torras, proposes a new method that first identifies the type of garment and then performs a search for the two grasping points that allow a robot to bring the garment to a known pose. The experiments conducted with real robots show that most of the errors are due to unsuccessful grasps and not to the localization of the grasping points, thus a more robust grasping strategy is required. $S I_{20}$ is similar to the work in [81] which makes use of a physics engine to create a training database. However, $S I_{20}$ aims at avoiding costly 


\subsection{Human Pose Estimation}

$S_{4}$ : The paper "A Very Simple Framework for 3D Human Pose Estimation Using a Single 2D Image: Comparison of Geometric Moments Descriptors" by Dieudinné Fabrice Atrevi, Damien Vivet, Bruno Emile and Florent Duculty, uses geometric moments to analyze the human silhouette from a single image. The proposed framework extracts the 3D human posture from a single 2D image in real time. The approach makes use of the learned correspondences between silhouettes and skeletons, extracted from synthetic 3D human models. The main contribution of this paper is the proposed technique to estimate 3D human motion via 3D synthesis software, which avoids the labour intensive manual data collection and annotation. Extensive experimental results on both synthetic and real-world datasets demonstrate the superior performance of the proposed framework compared with state-of-the-art methods. $S I_{4}$ is similar to the works in $[82,83]$ recovering $3 \mathrm{D}$ human pose from single 2D images. However $S I_{4}$ uses shape-from-silhouette method to find 3D pose from a single image, being robust even in the case of noisy silhouettes.

$S I_{12}$ : The paper "Generation of Human Depth Images with Body Part Labels for Complex Human Pose Recognition" by K. Nishi and J. Miura, develops a method for generating body-part annotated depth images of various body shapes and poses. The method is guided by a flexible human body model and a motion capture system. Based on the proposed method, the authors constructed a dataset of $10 \mathrm{~K}$ images with eight body types for various sitting poses. The effectiveness of the generated dataset is verified by solving the part-labeling tasks using a convolutional network (FCN). $S I_{12}$ extends the work in [84] from hand-level activities to finger-level hand activities analysis.

$S I_{15}$ : The paper " A Hybrid Framework for Automatic Joint Detection of Human Poses in Depth Frames" by Longbo Kong, Xiaohui Yuan, and Amar Man Maharjan, proposes a novel framework to detect joints automatically by using depth camera. The proposed method categorizes the joints into implicit or dominant joints, where implicit joints are the torso (i.e., neck and shoulders) and dominant joints are elbows and knees. In this framework, a loose skeleton 
model is used to locate implicit joints and data-driven method is applied to detect dominant joints. It uses a hierarchy of three CNNs with different levels of specialization, trained both with synthetic and real images. The results demonstrate that the proposed work can deliver stable and accurate detection results of joints. Overall, $S I_{15}$ combines a human body model and geodesic features of the human body together to detect and estimate the position of joints, achieving more accurate joint detection than related works in $[85,86]$.

\subsection{Deformable Shape Segmentation} tion on 3D Deformable Shapes" by Xupeng Wang, Ferdous Sohel, Mohammed Bennamoun, Yulan Guo and Hang Lei, detects a salient region, based on clustering of a data set in a scale space generated by an auto diffusion function. The proposed method is called Scale Space Clustering Evolution (SSCE). It consists of three parts: scale field construction, shape segmentation initialization and salient region detection. The auto diffusion function is used to extract shape features at multiple time scales. The initial segmentation of the shape is obtained using persistence-based clustering. The salient regions are detected during the evolution of the scale field. The experimental results obtained on popular datasets show a very promising performance of the proposed framework. SSCE inherits the merits of persistence-based clustering [87] and clustering assessment [88] for the benefit of salient region detection on 3D deformable shapes, and thus improving accuracy.

\subsection{Face Analysis}

$S I_{10}$ : The paper "Age and Gender Recognition in the Wild with Deep Attention” by Pau Rodriguez López, Guillem Cucurull Preixens, Josep M Gonfaus, Francesc Xavier Roca Marvá and Jordi González Sabaté, proposes a feedforward attention mechanism for age and gender classification. In this paper, a model that consists of an attention network is employed to discover the most

informative and reliable patches for age and gender classification. These patches 
are then further processed in a patch network in higher resolution to improve accuracy. With such attention mechanism, the model is able to discover the most informative and reliable parts in a face image even under deformation and occlusion. Experimental validation on the Adience, IoG and MORPH II dataset bemchmarks show that including attention mechanisms enhances the performance of CNNs in terms of robustness and accuracy. $S I_{10}$ is biologically inspired and benefits from the recent successes of attention mechanisms [89].

$S I_{17}$ : The paper "Gaussian Mixture 3D Morphable Face Model" by Paul Koppen, Zhen-Hua Feng, Josef Kittler, William Christmas, Xiao-Jun Wu, and He-Feng Yin, presents a Gaussian Mixture 3D Morphable Face Model (GM$3 \mathrm{DMM}$ ) to represent a global population of $3 \mathrm{D}$ faces as a mixture of Gaussian subpopulations. It extends the traditional 3DMM [90] naturally by adopting a shared covariance structure to mitigate small sample estimation problems associated with data in high dimensional spaces. Experiments in fitting the GM-3DMM to 2D face images to facilitate their geometric and photometric normalisation for pose and illumination invariant face recognition demonstrates the merit of the proposed multiple cohort 3D face model.

\section{Conclusion}

The aim of this guest editorial was to introduce this special issue on Articulated Motion and Deformable Object Recognition. The 20 papers accepted for the special issue cover four of the main subtopics of AMDO: human motion analysis (action/gesture), human pose estimation, deformable shape segmentation, and face analysis. The papers were introduced in the context of the recent developments in the field reviewed in this editorial.

585

Limitations and Challenges of AMDO. Although the accepted papers push the boundaries of the state of the art, there are still some limitations and challenges. First of all, there is a scope for exploring hybrid deep learning networks, as pioneered in $S I_{1}$, to capture spatial-temporal structure information more comprehensively. Second, the problem of fusing multiple modalities remains an 

dad, Gobierno de España), EPSRC/dstl supported projects EP/K014307/1 and EP/R018456/1, and EPSRC supported projects EP/N007743/1 and EP/P022529/1.

\section{References}

[1] W. Li, Z. Zhang, Z. Liu, Action recognition based on a bag of 3d points, Recognition Workshops, 2010, pp. 9-14. 
[2] S. Fothergill, H. Mentis, P. Kohli, S. Nowozin, Instructing people for training gestural interactive systems, in: Proceedings of the SIGCHI Conference on Human Factors in Computing Systems, ACM, 2012, pp. 1737-1746.

[3] J. Wang, Z. Liu, Y. Wu, J. Yuan, Mining actionlet ensemble for action recognition with depth cameras, in: Proceedings of the IEEE Conference on Computer Vision and Pattern Recognition, 2012, pp. 1290-1297.

[4] L. Xia, C. Chen, J. K. Aggarwal, View invariant human action recognition using histograms of 3d joints, in: Proceedings of the IEEE Conference on Computer Vision and Pattern Recognition Workshops, 2012, pp. 20-27.

[5] K. Yun, J. Honorio, D. Chattopadhyay, T. L. Berg, D. Samaras, Twoperson interaction detection using body-pose features and multiple instance learning, in: Computer Vision and Pattern Recognition Workshops (CVPRW), 2012 IEEE Computer Society Conference on, IEEE, 2012, pp. $28-35$.

[6] A. Shahroudy, J. Liu, T. Ng, G. Wang, Ntu rgb+d: A large scale dataset for $3 \mathrm{~d}$ human activity analysis, in: Proceedings of the IEEE Conference on computer vision and pattern recognition, 2016, pp. 1010-1019.

[7] Q. De Smedt, H. Wannous, J.-P. Vandeborre, Skeleton-based dynamic hand gesture recognition, in: Proceedings of the IEEE Conference on Computer Vision and Pattern Recognition Workshops, 2016, pp. 1-9.

[8] S. Escalera, X. Baró, J. Gonzalez, M. Á. Bautista, M. Madadi, M. Reyes, V. Ponce-López, H. J. Escalante, J. Shotton, I. Guyon, Chalearn looking at people challenge 2014: Dataset and results., in: ECCV Workshops, 2014, pp. $459-473$.

[9] V. Carletti, P. Foggia, G. Percannella, A. Saggese, M. Vento, Recognition of human actions from rgb-d videos using a reject option, in: International Conference on Image Analysis and Processing, Springer, 2013, pp. 436-445. 
[10] Y. Song, D. Demirdjian, R. Davis, Tracking body and hands for gesture

[12] R. Qiao, L. Liu, C. Shen, A. van den Hengel, Learning discriminative trajectorylet detector sets for accurate skeleton-based action recognition, Pattern Recognition 66 (2017) 202-212.

[13] C. Chen, K. Liu, N. Kehtarnavaz, Real-time human action recognition recognition: Natops aircraft handling signals database, in: Automatic Face \&amp; Gesture Recognition and Workshops (FG 2011), 2011 IEEE International Conference on, IEEE, 2011, pp. 500-506.

[11] Y. Yang, C. Deng, S. Gao, W. Liu, D. Tao, X. Gao, Discriminative multiinstance multitask learning for $3 \mathrm{~d}$ action recognition, IEEE Transactions on Multimedia 19 (3) (2017) 519-529.

based on depth motion maps, Journal of real-time image processing 12 (1) (2016) 155-163.

[14] X. Zhang, Y. Wang, M. Gou, M. Sznaier, O. Camps, Efficient temporal sequence comparison and classification using gram matrix embeddings on a riemannian manifold, in: Proceedings of the IEEE Conference on Computer Vision and Pattern Recognition, 2016, pp. 4498-4507.

[15] M. Liu, H. Liu, C. Chen, Enhanced skeleton visualization for view invariant human action recognition, Pattern Recognition 68 (2017) 346-362.

[16] Y. Hou, Z. Li, P. Wang, W. Li, Skeleton optical spectra based action recognition using convolutional neural networks, IEEE Transactions on Circuits and Systems for Video Technology.

[17] P. Wang, Z. Li, Y. Hou, W. Li, Action recognition based on joint trajectory maps using convolutional neural networks, in: Proceedings of the 2016 ACM on Multimedia Conference, ACM, 2016, pp. 102-106.

[18] A. Shahroudy, T.-T. Ng, Y. Gong, G. Wang, Deep multimodal feature analysis for action recognition in $\mathrm{rgb}+\mathrm{d}$ videos, IEEE Transactions on Pattern Analysis and Machine Intelligence. 
[19] Z. Luo, B. Peng, D.-A. Huang, A. Alahi, L. Fei-Fei, Unsupervised learning of long-term motion dynamics for videos.

[20] J. Wan, G. Guo, S. Z. Li, Explore efficient local features from rgb-d data for one-shot learning gesture recognition, IEEE transactions on pattern analysis and machine intelligence 38 (8) (2016) 1626-1639.

[21] S. Zhang, X. Liu, J. Xiao, On geometric features for skeleton-based action recognition using multilayer lstm networks, in: Applications of Computer Vision (WACV), 2017 IEEE Winter Conference on, IEEE, 2017, pp. 148157.

[22] D. C. Luvizon, H. Tabia, D. Picard, Learning features combination for human action recognition from skeleton sequences, Pattern Recognition Letters.

[23] M. Li, H. Leung, Graph-based approach for 3d human skeletal action recognition, Pattern Recognition Letters 87 (2017) 195-202.

[24] F. Ahmed, P. P. Paul, M. L. Gavrilova, Joint-triplet motion image and local binary pattern for $3 \mathrm{~d}$ action recognition using kinect, in: Proceedings of the 29th International Conference on Computer Animation and Social Agents, ACM, 2016, pp. 111-119.

[25] Q. Ke, S. An, M. Bennamoun, F. Sohel, F. Boussaid, Skeletonnet: Mining deep part features for 3-d action recognition, IEEE Signal Processing Letters 24 (6) (2017) 731-735.

[26] W. Zhu, C. Lan, J. Xing, W. Zeng, Y. Li, L. Shen, X. Xie, et al., Cooccurrence feature learning for skeleton based action recognition using regularized deep lstm networks., in: AAAI, Vol. 2, 2016, p. 8.

[27] C. Li, Y. Hou, P. Wang, W. Li, Joint distance maps based action recognition with convolutional neural networks, IEEE Signal Processing Letters 24 (5) (2017) 624-628. 
[28] N. Neverova, C. Wolf, G. Taylor, F. Nebout, Moddrop: adaptive multimodal gesture recognition, IEEE Transactions on Pattern Analysis and Machine Intelligence 38 (8) (2016) 1692-1706.

[29] E. P. Ijjina, K. M. Chalavadi, Human action recognition in rgb-d videos using motion sequence information and deep learning, Pattern Recognition 72 (2017) 504-516.

[30] L. Brun, G. Percannella, A. Saggese, M. Vento, Action recognition by using kernels on aclets sequences, Computer Vision and Image Understanding 144 (2016) 3-13.

[31] A. Joshi, C. Monnier, M. Betke, S. Sclaroff, Comparing random forest approaches to segmenting and classifying gestures, Image and Vision Computing 58 (2017) 86-95.

[32] J. Zhang, W. Li, P. O. Ogunbona, P. Wang, C. Tang, Rgb-d-based action recognition datasets: A survey, Pattern Recognition 60 (2016) 86-105.

[33] F. J. Perales, J. Santos-Victor, 8th articulated motion and deformable objects, in: 8th International Conference, AMDO 2014, 2014.

[34] F. J. Perales, J. Kittler, 9th articulated motion and deformable objects, in: 9th International Conference, AMDO 2016, 2016.

[35] S. Escalera, V. Athitsos, I. Guyon, Challenges in multi-modal gesture recognition, in: Gesture Recognition, Springer, 2017, pp. 1-60.

[36] M. Asadi-Aghbolaghi, A. Clapés, M. Bellantonio, H. J. Escalante, V. PonceLópez, X. Baró, I. Guyon, S. Kasaei, S. Escalera, Deep learning for action and gesture recognition in image sequences: A survey, in: Gesture Recognition, Springer, 2017, pp. 539-578.

[37] L. Sigal, A. O. Balan, M. J. Black, Humaneva: Synchronized video and motion capture dataset and baseline algorithm for evaluation of articulated human motion, International journal of computer vision 87 (1) (2010) 4-27. 
[38] F. Bogo, A. Kanazawa, C. Lassner, P. Gehler, J. Romero, M. J. Black, Keep it smpl: Automatic estimation of $3 \mathrm{~d}$ human pose and shape from a single image, in: European Conference on Computer Vision, Springer, 2016, pp. 561-578.

[39] M. Loper, N. Mahmood, J. Romero, G. Pons-Moll, M. J. Black, Smpl: A skinned multi-person linear model, ACM Transactions on Graphics (TOG) 34 (6) (2015) 248.

[40] X. Zhou, M. Zhu, S. Leonardos, K. G. Derpanis, K. Daniilidis, Sparseness meets deepness: 3d human pose estimation from monocular video, in: Proceedings of the IEEE Conference on Computer Vision and Pattern Recognition, 2016, pp. 4966-4975.

[41] B. Tekin, P. Marquez Neila, M. Salzmann, P. Fua, Learning to fuse 2d and $3 \mathrm{~d}$ image cues for monocular body pose estimation, in: International Conference on Computer Vision (ICCV), no. EPFL-CONF-230311, 2017.

[42] A. Elhayek, E. de Aguiar, A. Jain, J. Thompson, L. Pishchulin, M. Andriluka, C. Bregler, B. Schiele, C. Theobalt, Marconiconvnet-based markerless motion capture in outdoor and indoor scenes, IEEE transactions on pattern analysis and machine intelligence 39 (3) (2017) 501-514.

[43] P. Shilane, P. Min, M. Kazhdan, T. Funkhouser, The princeton shape benchmark, in: Shape modeling applications, 2004. Proceedings, IEEE, 2004, pp. 167-178.

[44] X. Chen, A. Golovinskiy, T. Funkhouser, A benchmark for 3d mesh segmentation, in: Acm transactions on graphics (tog), Vol. 28, ACM, 2009, p. 73 .

[45] T. Le, G. Bui, Y. Duan, A multi-view recurrent neural network for 3d mesh segmentation, Computers \& Graphics. 
[46] Z. Shu, C. Qi, S. Xin, C. Hu, L. Wang, Y. Zhang, L. Liu, Unsupervised $3 \mathrm{~d}$ shape segmentation and co-segmentation via deep learning, Computer Aided Geometric Design 43 (2016) 39-52.

[47] D. Riccio, G. Tortora, M. De Marsico, H. Wechsler, Egaethnicity, gender and age, a pre-annotated face database, in: Biometric Measurements and Systems for Security and Medical Applications (BIOMS), 2012 IEEE Workshop on, IEEE, 2012, pp. 1-8.

[48] http://www.face-rec.org/databases/.

[49] M. Castrillón-Santana, M. De Marsico, M. Nappi, D. Riccio, Meg: Texture operators for multi-expert gender classification, Computer Vision and Image Understanding 156 (2017) 4-18.

[50] A. Dantcheva, P. Elia, A. Ross, What else does your biometric data reveal? a survey on soft biometrics, IEEE Transactions on Information Forensics and Security 11 (3) (2016) 441-467.

[51] E. Eidinger, R. Enbar, T. Hassner, Age and gender estimation of unfiltered faces, IEEE Transactions on Information Forensics and Security 9 (12) (2014) 2170-2179.

[52] A. C. Gallagher, T. Chen, Understanding images of groups of people, in: Computer Vision and Pattern Recognition, 2009. CVPR 2009. IEEE Conference on, IEEE, 2009, pp. 256-263.

[53] K. Ricanek, T. Tesafaye, Morph: A longitudinal image database of normal adult age-progression, in: Automatic Face and Gesture Recognition, 2006. FGR 2006. 7th International Conference on, IEEE, 2006, pp. 341-345.

[54] Z. Tan, J. Wan, Z. Lei, R. Zhi, G. Guo, S. Z. Li, Efficient group-n encoding and decoding for facial age estimation, IEEE Transactions on Pattern Analysis and Machine Intelligence (TPAMI), 2017. 
[55] Y. Chen, Z. Tan, A. P. Leung, J. Wan, J. Zhang, Multi-region ensemble convolutional neural networks for high-accuracy age estimation.

780

[61] Y. Sun, Y. Chen, X. Wang, X. Tang, Deep learning face representation by joint identification-verification, in: Advances in neural information processing systems, 2014, pp. 1988-1996.

[62] Y. Sun, D. Liang, X. Wang, X. Tang, Deepid3: Face recognition with very deep neural networks, arXiv preprint arXiv:1502.00873.

[63] C. A. Corneanu, M. O. Simon, J. F. Cohn, S. E. Guerrero, Survey on rgb, 3d, thermal, and multimodal approaches for facial expression recognition: History, trends, and affect-related applications, IEEE transactions on pattern analysis and machine intelligence 38 (8) (2016) 1548-1568. 
[64] E. Agustsson, R. Timofte, S. Escalera, X. Baro, I. Guyon, R. Rothe, Apparent and real age estimation in still images with deep residual regressors on appa-real database, in: Automatic Face and Gesture Recognition, 2006. FGR 2006. 7th International Conference on, 2017.

[65] J. Donahue, L. Anne Hendricks, S. Guadarrama, M. Rohrbach, S. Venugopalan, K. Saenko, T. Darrell, Long-term recurrent convolutional networks for visual recognition and description, in: Proceedings of the IEEE conference on computer vision and pattern recognition, 2015, pp. 26252634 .

[66] K. Schindler, L. Van Gool, Action snippets: How many frames does human action recognition require?, in: Computer Vision and Pattern Recognition, 2008. CVPR 2008. IEEE Conference on, IEEE, 2008, pp. 1-8.

[67] A. Srivastava, E. Klassen, S. H. Joshi, I. H. Jermyn, Shape analysis of elastic curves in euclidean spaces, IEEE Transactions on Pattern Analysis and Machine Intelligence 33 (7) (2011) 1415-1428.

[68] M. Braham, M. Van Droogenbroeck, Deep background subtraction with scene-specific convolutional neural networks, in: Systems, Signals and Image Processing (IWSSIP), 2016 International Conference on, IEEE, 2016, pp. 1-4.

[69] J. Wan, Q. Ruan, W. Li, S. Deng, One-shot learning gesture recognition from rgb-d data using bag of features, The Journal of Machine Learning Research 14 (1) (2013) 2549-2582.

[70] M. S. Ryoo, Human activity prediction: Early recognition of ongoing activities from streaming videos, in: Computer Vision (ICCV), 2011 IEEE International Conference on, IEEE, 2011, pp. 1036-1043.

${ }_{830}$ [71] X. Zhao, S. Wang, X. Li, H. L. Zhang, Online action recognition by template matching, in: International Conference on Health Information Science, Springer, 2013, pp. 269-272. 
[72] A. Galata, N. Johnson, D. Hogg, Learning variable-length markov models of behavior, Computer Vision and Image Understanding 81 (3) (2001) 398413.

[73] Y. Du, Y. Fu, L. Wang, Skeleton based action recognition with convolutional neural network, in: Pattern Recognition (ACPR), 2015 3rd IAPR Asian Conference on, IEEE, 2015, pp. 579-583.

[74] D. C. Mocanu, H. B. Ammar, D. Lowet, K. Driessens, A. Liotta, G. Weiss, K. Tuyls, Factored four way conditional restricted boltzmann machines for activity recognition, Pattern Recognition Letters 66 (2015) 100-108.

[75] Y. Dai, H. Li, M. He, A simple prior-free method for non-rigid structurefrom-motion factorization, International Journal of Computer Vision 107 (2) (2014) 101-122.

${ }_{845}$ [76] M. Lee, J. Cho, C.-H. Choi, S. Oh, Procrustean normal distribution for non-rigid structure from motion, in: Proceedings of the IEEE Conference on Computer Vision and Pattern Recognition, 2013, pp. 1280-1287.

[77] S. He, Q. Yang, R. W. Lau, J. Wang, M.-H. Yang, Visual tracking via locality sensitive histograms, in: Proceedings of the IEEE Conference on Computer Vision and Pattern Recognition, 2013, pp. 2427-2434.

[78] M. Danelljan, F. Shahbaz Khan, M. Felsberg, J. Van de Weijer, Adaptive color attributes for real-time visual tracking, in: Proceedings of the IEEE Conference on Computer Vision and Pattern Recognition, 2014, pp. 10901097.

[79] L. Zhou, W. Li, P. Ogunbona, Learning a pose lexicon for semantic action recognition, in: Multimedia and Expo (ICME), 2016 IEEE International Conference on, IEEE, 2016, pp. 1-6.

[80] M. Meshry, M. E. Hussein, M. Torki, Linear-time online action detection from 3d skeletal data using bags of gesturelets, in: Applications of Com- 
puter Vision (WACV), 2016 IEEE Winter Conference on, IEEE, 2016, pp. $1-9$.

[81] Y. Li, D. Xu, Y. Yue, Y. Wang, S.-F. Chang, E. Grinspun, P. K. Allen, Regrasping and unfolding of garments using predictive thin shell modeling, in: Robotics and Automation (ICRA), 2015 IEEE International Conference on, IEEE, 2015, pp. 1382-1388.

[82] D. F. Fouhey, V. Delaitre, A. Gupta, A. A. Efros, I. Laptev, J. Sivic, People watching: Human actions as a cue for single view geometry, International journal of computer vision 110 (3) (2014) 259-274.

[83] Y. Yang, D. Ramanan, Articulated pose estimation with flexible mixturesof-parts, in: Computer Vision and Pattern Recognition (CVPR), 2011 IEEE Conference on, IEEE, 2011, pp. 1385-1392.

[84] J. Wang, Z. Liu, Y. Wu, Learning actionlet ensemble for 3d human action recognition, in: Human Action Recognition with Depth Cameras, Springer, 2014, pp. 11-40.

${ }_{875}[85]$ K. Buys, C. Cagniart, A. Baksheev, T. De Laet, J. De Schutter, C. Pantofaru, An adaptable system for rgb-d based human body detection and pose estimation, Journal of visual communication and image representation 25 (1) (2014) 39-52.

[86] K. Nishi, J. Miura, Generation of human depth images with body part labels for complex human pose recognition, Pattern Recognition.

[87] F. Chazal, L. J. Guibas, S. Y. Oudot, P. Skraba, Persistence-based clustering in riemannian manifolds, Journal of the ACM (JACM) 60 (6) (2013) 41.

[88] B. Rieck, H. Leitte, Exploring and comparing clusterings of multivariate data sets using persistent homology, in: Computer Graphics Forum, Vol. 35, Wiley Online Library, 2016, pp. 81-90. 
[89] V. Mnih, N. Heess, A. Graves, et al., Recurrent models of visual attention, in: Advances in neural information processing systems, 2014, pp. 22042212.

[90] V. Blanz, T. Vetter, A morphable model for the synthesis of 3d faces, in: Proceedings of the 26th annual conference on Computer graphics and interactive techniques, ACM Press/Addison-Wesley Publishing Co., 1999, pp. 187-194.

[91] P. Mettes, C. G. Snoek, Spatial-aware object embeddings for zero-shot localization and classification of actions, ICCV (2017) 4443-4452. 